\title{
Using Household Economic Survey Data to Assess Food Expenditure Patterns and Trends in a Country with Notable Health Inequities
}

Nhung Nghiem ( $\nabla$ nhung.nghiem@otago.ac.nz )

University of Otago, Wellington https://orcid.org/0000-0003-0078-4549

\section{Andrea Teng}

University of Otago

Christine Cleghorn

University of Otago

Christina McKerchar

University of Otago

Nick Wilson

University of Otago

\section{Research}

Keywords: Food expenditure, dietary risk factors, household economic survey, New Zealand

Posted Date: November 8th, 2021

DOI: https://doi.org/10.21203/rs.3.rs-1048731/v1

License: (c) (1) This work is licensed under a Creative Commons Attribution 4.0 International License. Read Full License 


\section{Abstract}

\section{Background}

Dietary factors are one of the most important risk factors for health loss worldwide, however there is limited information on dietary trends in Aotearoa New Zealand (NZ) and whether inequities in dietary patterns are changing.

\section{Methods}

We extracted data from the Household Economic Survey (HES), which was designed to provide information on impacts of policy-making in NZ. Three HES waves in 2006/07, 2009/10 and 2012/13 $(n=9030)$ were used to examine the trends in household expenditure for key food groups by income-level and ethnicity, including food group expenditure (absolute amounts and as a proportion of total food expenditure, inflation adjusted).

\section{Results}

Overall, total household food expenditure per capita (CPI-adjusted) increased by $0.38 \%$ annually over this period (albeit not statistically significant). In absolute terms, low-income households spent around three quarters of what high-income households spent on food per capita. High-income households experienced a greater increase in expenditure on nuts and seeds (increasing the gap) and a greater reduction in expenditure on processed meat (reducing the gap because expenditure remained higher than in lowincome households).

There was increased expenditure over time on fruit and vegetables (NZ\$273 per capita per year), nuts and seeds, and healthy foods (NZ\$507) in Māori (Indigenous) households with little variations in non-Māori households. As a result, the relative health-related gap in food expenditure between Māori and non-Māori households declined over time. But there was little change in processed meat expenditure for Māori households and expenditure on less healthy foods also increased over time.

\section{Conclusions}

HES data were useful for understanding trends in food expenditure patterns, in the absence of nutrition survey data. Potentially positive expenditure trends for Māori were identified, which could indicate a shift towards healthier diets. However, food expenditure inequities in processed meat and less healthy foods by ethnicity and income continue to be substantial. Public policies that aim to support healthy diets for all could involve changes to the food environment and government support for culturally appropriate Māori-led interventions.

\section{Introduction}


Dietary risk factors are one of the most important risk factors for non-communicable diseases (NCDs) worldwide, accountable for 11 million premature deaths and the loss of 225 million disability-adjusted life years (DALYs) in 2017. ${ }^{(1)}$ These risk factors contribute to cardiovascular diseases (CVD), diabetes, and cancer; which are among the top leading causes of deaths globally. ${ }^{(2)}$ Household food expenditure surveys are increasingly used to monitor changes in dietary patterns internationally as individual nutrition survey data are often lacking and such nutrition surveys are expensive. ${ }^{(3-7)}$ Employing food expenditure data, studies have suggested that urbanisation, industrialisation and globalisation among other factors have shifted dietary patterns towards more processed foods. ${ }^{(8-11)}$ Food-insecurity is also highly correlated with total household food expenditure, $(12,13)$ and low-income households often lack access to nutritious foods. ${ }^{(14)}$

In Aotearoa New Zealand (NZ), the NZ Burden of Disease Study shows that nutrition and obesity factors contribute to $18.6 \%$ of total health loss (in DALYs). In addition, diet and obesity related diseases are unequally distributed by ethnicity and deprivation, with Māori, Pasifika and groups with low socioeconomic position at a higher risk of having obesity ${ }^{(15)}$ and NCDs. ${ }^{(16)}$ Much of this health loss and premature death could be prevented by improved diet and addressing the obesogenic environment that encourages unhealthy nutrition. Dietary patterns that are high in sodium, low in fruits and vegetables, low in nuts and seeds, high in processed meat and high in sugar-sweetened beverages are the major risk factors for NCDs including CVD, diabetes and cancer. $(1,17)$

Despite the relative importance of dietary risk factors in generating health outcomes, the most recent Adult Nutrition Survey in NZ was over a decade ago (2008/09) and for children it was around two decades ago (2002). ${ }^{(18)}$ For this reason, there are no recent representative data on trends in dietary patterns in NZ (but note that the NZ Health Survey included food frequency questions in 2019/20), nor changes in the distribution of diet by social factors.

Furthermore, different data sources have different strengths and weaknesses. Individual dietary intake includes many sources of food but often relies on the participants' memory. Trends in household food expenditure do not directly indicate individual diet, but are particularly relevant to concerns about food security and the cost of healthy food, particularly for low-income households (eg, the proportion of income spent on food). Expenditure data can also be used to examine changes in types of food bought over time.

The linkage of the NZ Household Economic Survey (HES) data $(2006 / 07,2009 / 10,2012 / 13)^{(19)}$ creates repeated cohorts of nationally representative data. The HES contains detailed information about household food expenditure, alcohol expenditure, tobacco expenditure, and other non-food household expenditure; and is implemented every three years. ${ }^{(20)}$ HES can also contribute information on food consumption patterns. ${ }^{(4,21)}$

We therefore aimed to explore the trends and social patterns in NZ household dietary expenditure using national representative linked HES data in NZ. 


\section{Methods}

We used data from three HES waves $(2006 / 07,2009 / 10,2012 / 13)$ with a total of 9030 households. ${ }^{(19,22)}$ These samples were randomly drawn from the total NZ resident population. The HES comprises questionnaires on the household, expenditure, income, and a two-week expenditure diary. Household expenditure includes: food ( 500 food items), alcohol, tobacco, transportation, housing, health, education, recreation and culture, and other goods and services. There is a strong correlation between what individuals report about individual income in the HES and their actual income recorded in the Inland Revenue data. ${ }^{(22)}$

Three specific food groups were examined: fruit and vegetables (including frozen), nuts and seeds and processed meat, as per Global Burden of Disease (GBD) Study 2017 data, ${ }^{(1)}$ Ni Mhurchu et al (2015)(23) which used HES data, and our previous work. ${ }^{(24)}$ These food groups were selected because they are major risk factors for $\mathrm{CVD}^{(1)}$ and can be categorised in the HES. An overall healthy food group was defined using the nutrient-profiling criteria as per Waterlander et al ${ }^{25,26)}$ and included all fresh fruit and vegetables (plus frozen fruit and vegetables), fresh seafood, nuts and seeds, whole grains, milk, legumes and bottled water. A less healthy group was defined as all the remaining food and beverages, such as sugar-sweetened beverages, snack food such as potato chips, confectionary, and takeaway foods.

The main outcomes of interest for each food group were expenditure per person per year (2013 NZ\$, annual values were provided by the data provider Stats NZ), expenditure as a proportion of total food expenditure, and expenditure relative to total income, all obtained from the HES data. We deflated income and expenditure to get comparable measurements across HES waves. We calculated mean expenditure and its standard error (SE) for total food and each food sub-group in each HES wave. Expenditure by average household income-level per person (high- and low-income were defined as above and below the median for the HES survey respectively) and by household ethnicity (whether any household members self-identified as Māori [Indigenous population] or not) were also calculated. Expenditure trends, relative risks and significance levels were estimated using linear regressions, employing survey weights and adjusting for sampling structure. Independent variables for these linear regression models were survey year and either household income-level per person or household ethnicity.

Data were extracted using SQL version v.18.8, and were further processed and analysed in R using the 'survey' package, version R.3.6.0.

\section{Results}

There was a total of 9030 household survey participants evenly distributed across three HESs (2901 in 2006/07, 3126 in 2009/10, 3003 in 2012/13). Data on food expenditure was not available for $0.76 \%$ of households and they were excluded from the analysis; and $0.63 \%$ households had no income and so were unable to be included for the expenditure as a proportion of income outcome measure. Mean household income of the total sample was NZ\$37,700. 
Māori households accounted for around $17 \%$ of the total sample (1,520 households). Māori households across all cohorts had significant differences to non-Māori households in income, age, household size and the number of households with children. Māori households had a lower mean income (NZ \$30,400 per capita) compared to non-Māori (NZ\$39,200 per capita). Māori households spent less on food per capita NZ\$3,490 (11.5\% of total income) compared to NZ\$4,230 (10.8\% of total income) for non-Māori. Māori households tended to have more people, with a medium size of 3.09 compared to 2.43 for nonMāori and had a greater percentage of households with children; $48.8 \%$ compared to $34.5 \%$ non-Maori households with children (see Appendix A). For further characteristics of the survey samples see Table 1. 
Table 1

Characteristics of repeated New Zealand Household Economic Survey samples

\section{Specific metrics}

Total sample $^{\mathrm{a}}$

\section{General characteristics}

Households sample size (n)

9,030

2,900

3,130

3,000

Households included in this study (n)

8,910

2,850

3,090

2,960

Households excluded because they had no food expenditure data $(n, \%)$

69

24

12

$(0.76 \%)$

$(0.83 \%) \quad(0.38 \%)$

30

Households excluded because they had no income data (zero or negative income)

57

$(0.63 \%)$

24

$(0.83 \%)$

21

$(0.67 \%)$

9

Households members ( $\mathrm{n}$ )

22,700

37.3

Mean age (years)

Household composition

Households with children (n)

3,340

$(37.5 \%)$

1,080

$(37.7 \%)$

1,200

$(38.8 \%)$

1,070

$(36 \%)$

Households with members aged 65 or more years old (n)

Mean household size

1,760

$(19.7 \%)$

507

579

(18.7\%)

672

(22.7\%)

2.54

2.57

2.59

2.47

Sex

Male (n)

Female (n)

Household income (NZ\$ in 2013 value/year) per capita

Mean

Median

Low-income: - mean

- median

High-income: - mean

- median
37,700

28,400

24,900

20,900

57,900

47,300
37,500

28,200

24,500

20,800

57,100

47,100
3,850

3,510

4,160

3,810
38.9 


\begin{tabular}{|c|c|c|c|c|}
\hline Specific metrics & $\begin{array}{l}\text { Total } \\
\text { sample }^{a}\end{array}$ & $2006 / 07$ & $2009 / 10$ & 2012/13 \\
\hline Māori income: - mean & 30,400 & 32,500 & 27,500 & 31,500 \\
\hline - median & 23,200 & 23,400 & 22,800 & 23,300 \\
\hline Non-Māori income: - mean & 39,200 & 38,500 & 39,100 & 39,900 \\
\hline - median & 29,700 & 29,300 & 30,100 & 29,500 \\
\hline \multicolumn{5}{|l|}{ Household ethnicity } \\
\hline Households with any Māori member (n) & 1,520 & 471 & 552 & 492 \\
\hline Households with no Māori members (n) & 7,390 & 2,380 & 2,540 & 2,470 \\
\hline
\end{tabular}

\section{Expenditure trends}

Annual household food expenditure per capita (in 2013 NZ\$) by food group for three HES waves: 2006/07, 2009/10, and 2013/13 are described in Table 2.

Overall trends: Total household food expenditure was increasing slightly from an average of NZ\$3,990/person (se: 64.7) in 2006/07 to NZ\$4,080 (se: 60.9) in 2012/13, after adjusting for inflation. Relatively, total food expenditure (out of all expenditure) appeared to increase by $1.13 \% /$ three years or $0.38 \%$ per year between $2006-2012$. Expenditure on fruit and vegetables (-1.28\% change), processed meat $(-2.01 \%)$, and healthy food (-1.71\%), appeared to decrease slightly over time. Expenditure on less healthy foods appeared to increase by $1.79 \%$, and nuts and seeds by $5.85 \%$. These estimated trends in expenditure had wide uncertainty (se $>50 \%$ of the mean) and were not statistically significant.

Trends by income: Total annual food expenditure for low-income households appeared to increase by $3.11 \%$ to NZ\$3,580 (se: 64.2 ) in 2012/13, whereas that figure for high-income households remained stable at NZ\$4,840 (se: 97.7). However, there was a peak in low-income household spending in 2009/10, and at the same time a dip in high-income household spending, which are masked when considering linear trend results. Both types of households appeared to slightly reduce their expenditure on fruit and vegetables, and on healthy foods. Expenditure on nuts and seeds appeared to increase more in highincome (8.33\%, se: 5.08$)$ than low-income households (3.70\%, se: 5.85$)$. There was a reduction in highincome household expenditure on processed meat over the years by $-4.20 \%$ (se: 2.37 ) not seen in lowincome households $(0.00 \%$ change, se: 2.22$)$. 
Trends by ethnic group: Total food expenditure for Māori households increased by $7.82 \%$ to NZ\$3,750 (se: 129.8) in 2012/13, but that for non-Māori households slightly reduced (albeit not statistically significant). Māori households increased their expenditure on fruit and vegetables (9.34\%, se: 4.14$)$, nuts and seeds $(25.0 \%$, se: 12.3$)$, but there was little change in expenditure on processed meat $(-1.05 \%$, se: 3.72). Māori households appeared to increase spending on healthy foods ( $4.34 \%$, se: $3.77 \%)$, whereas non-Māori households decreased their spending on healthy foods (-2.69\%, se: $1.35 \%)$. Further details of these food expenditures are provided in Table 2. 
Table 2

Annual expenditure per capita (in 2013 NZ\$) by food group, income-level and ethnicity in three HES waves: $2006 / 07,2009 / 10$, and 2013/13. ${ }^{a}$

\begin{tabular}{|c|c|c|c|c|c|}
\hline Food group & $\begin{array}{l}\text { Population } \\
\text { group }\end{array}$ & $\begin{array}{l}2006 / 07 \\
(2013 \\
\text { NZS, se) }\end{array}$ & $\begin{array}{l}2009 / 10 \\
(2013 \\
\text { NZ\$, se) }\end{array}$ & $\begin{array}{l}2012 / 13 \\
(2013 \\
\text { NZS, se) }\end{array}$ & $\begin{array}{l}\text { Relative change in food } \\
\text { expenditure over the survey } \\
\text { interval or every three years (\%: } \\
\text { mean (se)) }\end{array}$ \\
\hline \multirow[t]{5}{*}{ All food } & Total sample & $\begin{array}{l}3990 \\
(64.6)\end{array}$ & $\begin{array}{l}4130 \\
(49.1)\end{array}$ & $\begin{array}{l}4080 \\
(60.9)\end{array}$ & $1.13(1.04)^{b}$ \\
\hline & $\begin{array}{l}\text { Low-income } \\
\text { (less than } \\
\text { median) }\end{array}$ & $\begin{array}{l}3370 \\
(62.5)\end{array}$ & $\begin{array}{l}3740 \\
(67.5)\end{array}$ & $\begin{array}{l}3580 \\
(64.2)\end{array}$ & $3.11(1.33)$ \\
\hline & $\begin{array}{l}\text { High-income } \\
\text { (more than } \\
\text { median) }\end{array}$ & $\begin{array}{l}4840 \\
(1030)\end{array}$ & $\begin{array}{l}4720 \\
(84.2)\end{array}$ & $\begin{array}{l}4840 \\
(97.7)\end{array}$ & $0.02(1.47)$ \\
\hline & Māori & $\begin{array}{l}3240 \\
(116)\end{array}$ & $\begin{array}{l}3420 \\
(131)\end{array}$ & $\begin{array}{l}3750 \\
(129)\end{array}$ & $7.82(2.69)$ \\
\hline & Non-Māori & $\begin{array}{l}4170 \\
(69.7)\end{array}$ & $\begin{array}{l}4310 \\
(55)\end{array}$ & $\begin{array}{l}4160 \\
(68.7)\end{array}$ & $-0.12(1.17)$ \\
\hline \multirow[t]{5}{*}{$\begin{array}{l}\text { Fruit and } \\
\text { vegetables }\end{array}$} & Total sample & $\begin{array}{l}437 \\
(9.61)\end{array}$ & $\begin{array}{l}406 \\
(8.10)\end{array}$ & $\begin{array}{l}425 \\
(9.21)\end{array}$ & $-1.28(1.50)^{\mathrm{b}}$ \\
\hline & Low-income & $\begin{array}{l}399 \\
(11.3)\end{array}$ & $\begin{array}{l}378 \\
(10.9)\end{array}$ & $\begin{array}{l}393 \\
(10.6)\end{array}$ & $-0.75(1.95)$ \\
\hline & High-income & $\begin{array}{l}490 \\
(14.8)\end{array}$ & $\begin{array}{l}449 \\
(11.8)\end{array}$ & $\begin{array}{l}474 \\
(12.7)\end{array}$ & $-1.63(2.00)$ \\
\hline & Māori & $\begin{array}{l}273 \\
(14.9)\end{array}$ & $\begin{array}{l}275 \\
(13.3)\end{array}$ & $\begin{array}{l}324 \\
(16.9)\end{array}$ & $9.34(4.14)$ \\
\hline & Non-Māori & $\begin{array}{l}477 \\
(10.8)\end{array}$ & $\begin{array}{l}440 \\
(9.25)\end{array}$ & $\begin{array}{l}450 \\
(10.4)\end{array}$ & $-2.83(1.57)$ \\
\hline \multirow[t]{4}{*}{$\begin{array}{l}\text { Nuts and } \\
\text { seeds }\end{array}$} & Total sample & $\begin{array}{l}31 \\
(1.79)\end{array}$ & $\begin{array}{l}36 \\
(2.01)\end{array}$ & $\begin{array}{l}34 \\
(1.69)\end{array}$ & $5.85(3.57)^{b}$ \\
\hline & Low-income & $\begin{array}{l}27 \\
(2.37)\end{array}$ & $\begin{array}{l}31 \\
(2.77)\end{array}$ & $\begin{array}{l}29 \\
(2.09)\end{array}$ & $3.70(5.85)$ \\
\hline & High-income & $\begin{array}{l}36 \\
(2.49)\end{array}$ & $\begin{array}{l}43 \\
(3.09)\end{array}$ & $\begin{array}{l}42 \\
(2.68)\end{array}$ & $8.33(5.08)$ \\
\hline & Māori & $\begin{array}{l}14 \\
(2.02)\end{array}$ & $\begin{array}{l}18 \\
(2.92)\end{array}$ & $\begin{array}{l}21 \\
(2.78)\end{array}$ & $25.0(12.30)$ \\
\hline
\end{tabular}

Note: ${ }^{a}$ Numbers may not add up exactly as they were randomly rounded to meet confidentiality requirements. ${ }^{b}$ Values in this column were derived using linear regressions with the survey year as the only independent variable, but no changes were statistically significant. All values in this Table were calculated or estimated using survey weights. 


\begin{tabular}{|c|c|c|c|c|c|}
\hline \multirow[t]{2}{*}{ Food group } & \multirow{2}{*}{$\begin{array}{l}\text { Population } \\
\text { group }\end{array}$} & \multirow{2}{*}{$\begin{array}{l}2006 / 07 \\
(2013 \\
\text { NZ\$, se) }\end{array}$} & \multirow{2}{*}{$\begin{array}{l}2009 / 10 \\
(2013 \\
\text { NZ\$, se) }\end{array}$} & \multirow{2}{*}{$\begin{array}{l}2012 / 13 \\
(2013 \\
\text { NZ\$, se) }\end{array}$} & \multirow{2}{*}{$\begin{array}{l}\text { Relative change in food } \\
\text { expenditure over the survey } \\
\text { interval or every three years (\%: } \\
\text { mean (se)) }\end{array}$} \\
\hline & & & & & \\
\hline & Non-Māori & $\begin{array}{l}35 \\
(2.07)\end{array}$ & $\begin{array}{l}40 \\
(2.34)\end{array}$ & $\begin{array}{l}38 \\
(1.96)\end{array}$ & $4.29(4.07)$ \\
\hline \multirow[t]{5}{*}{$\begin{array}{l}\text { Processed } \\
\text { meat }\end{array}$} & Total sample & $\begin{array}{l}204 \\
(4.63)\end{array}$ & $\begin{array}{l}199 \\
(4.70)\end{array}$ & $\begin{array}{l}196 \\
(4.81)\end{array}$ & $-2.01(1.62)^{\mathrm{b}}$ \\
\hline & Low-income & $\begin{array}{l}189 \\
(6.07)\end{array}$ & $\begin{array}{l}191 \\
(6.76)\end{array}$ & $\begin{array}{l}189 \\
(5.78)\end{array}$ & $0.00(2.22)$ \\
\hline & High-income & $\begin{array}{l}226 \\
(7.24)\end{array}$ & $\begin{array}{l}211 \\
(6.52)\end{array}$ & $\begin{array}{l}207 \\
(7.92)\end{array}$ & $-4.20(2.37)$ \\
\hline & Māori & $\begin{array}{l}191 \\
(9.98)\end{array}$ & $\begin{array}{l}186 \\
(10.9)\end{array}$ & $\begin{array}{l}187 \\
(10.1)\end{array}$ & $-1.05(3.72)$ \\
\hline & Non-Māori & $\begin{array}{l}208 \\
(5.34)\end{array}$ & $\begin{array}{l}202 \\
(5.36)\end{array}$ & $\begin{array}{l}198 \\
(5.57)\end{array}$ & $-2.40(1.85)$ \\
\hline \multirow[t]{5}{*}{$\begin{array}{l}\text { Healthy } \\
\text { foods }\end{array}$} & Total sample & $\begin{array}{l}741 \\
(14.6)\end{array}$ & $\begin{array}{l}716 \\
(12.2)\end{array}$ & $\begin{array}{l}715 \\
(13.1)\end{array}$ & $-1.71(1.29)^{b}$ \\
\hline & Low-income & $\begin{array}{l}689 \\
(16.7)\end{array}$ & $\begin{array}{l}678 \\
(15.7)\end{array}$ & $\begin{array}{l}677 \\
(15.7)\end{array}$ & $-0.87(1.67)$ \\
\hline & High-income & $\begin{array}{l}814 \\
(22.1)\end{array}$ & $\begin{array}{l}774 \\
(19.2)\end{array}$ & $\begin{array}{l}774 \\
(18.9)\end{array}$ & $-2.46(1.79)$ \\
\hline & Māori & $\begin{array}{l}507 \\
(25.3)\end{array}$ & $\begin{array}{l}500 \\
(20.3)\end{array}$ & $\begin{array}{l}551 \\
(28.6)\end{array}$ & 4.34 (3.77) \\
\hline & Non-Māori & $\begin{array}{l}798 \\
(16.1)\end{array}$ & $\begin{array}{l}770 \\
(13.9)\end{array}$ & $\begin{array}{l}755 \\
(14.3)\end{array}$ & $-2.69(1.35)$ \\
\hline \multirow{5}{*}{$\begin{array}{l}\text { Remaining } \\
\text { less healthy } \\
\text { foods }\end{array}$} & Total sample & $\begin{array}{l}3250 \\
(56.4)\end{array}$ & $\begin{array}{l}3410 \\
(43.8)\end{array}$ & $\begin{array}{l}3360 \\
(54.1)\end{array}$ & $1.79(1.11)^{b}$ \\
\hline & Low-income & $\begin{array}{l}2680 \\
(52.8)\end{array}$ & $\begin{array}{l}3060 \\
(60.5)\end{array}$ & $\begin{array}{l}2910 \\
(58.0)\end{array}$ & $4.14(1.46)$ \\
\hline & High-income & $\begin{array}{l}4020 \\
(91.4)\end{array}$ & $\begin{array}{l}3950 \\
(74.7)\end{array}$ & $\begin{array}{l}4070 \\
(87.4)\end{array}$ & $0.52(1.57)$ \\
\hline & Māori & $\begin{array}{l}2730 \\
(101)\end{array}$ & $\begin{array}{l}2920 \\
(121)\end{array}$ & $\begin{array}{l}3200 \\
(122)\end{array}$ & $8.48(2.91)$ \\
\hline & Non-Māori & $\begin{array}{l}3370 \\
(60.9)\end{array}$ & $\begin{array}{l}3540 \\
(48.8)\end{array}$ & $\begin{array}{l}3400 \\
(60.5)\end{array}$ & $0.49(1.27)$ \\
\hline \multicolumn{6}{|c|}{$\begin{array}{l}\text { Note: }{ }^{a} \text { Numbers may not add up exactly as they were randomly rounded to meet confidentiality } \\
\text { requirements. }{ }^{b} \text { Values in this column were derived using linear regressions with the survey year as } \\
\text { the only independent variable, but no changes were statistically significant. All values in this Table } \\
\text { were calculated or estimated using survey weights. }\end{array}$} \\
\hline
\end{tabular}


Table 3 compares food expenditure by income-level and household ethnicity (relative risks) in each wave. Low-income and Māori households spent less money on all food categories in all years compared to high-income and non-Māori households respectively, and almost all of these differences were statistically significant.

The gap in expenditure between low- and high-income households (where high-income households spend more) increased over time for nuts and seeds and decreased over time for processed meat. Changes were less clear for other food groups.

The gap in expenditure between Māori and non-Māori households appeared to decline over time for fruit and vegetables, nuts and seeds, and healthy food. The gaps remaining, however, were wide and significant (with Māori households spending $72 \%, 55 \%$, and $73 \%$ respectively of what non-Māori households spent in 2012/13). Similarly, Māori households in 2006 reported a lower expenditure on less healthy foods (81\%) than non-Māori, however expenditure increased over time to become $94 \%$ (Cl: 86 102) of the level of non-Māori households in 2012-13. 
Table 3

Relative risks ${ }^{a}(\%)$ in household food purchase expenditure for low-income as proportion of high-income, and Māori as proportion of non-Māori households, Household Economic Surveys 2006/07, 2009/10, and 2012/13.

\begin{tabular}{|c|c|c|c|c|}
\hline \multirow[t]{2}{*}{ Food group } & $\begin{array}{l}\text { Population group (as } \\
\text { proportion of comparator) }\end{array}$ & $\begin{array}{l}\text { Relative risks } \\
\text { in } 2006 / 07\end{array}$ & $\begin{array}{l}\text { Relative risks } \\
\text { in } 2009 / 10\end{array}$ & $\begin{array}{l}\text { Relative risks } \\
\text { in } 2012 / 13\end{array}$ \\
\hline & & $\begin{array}{l}(\%: \text { mean } \\
(\text { se)) }\end{array}$ & $\begin{array}{l}(\%: \text { mean } \\
(\mathrm{se}))\end{array}$ & $\begin{array}{l}(\%: \text { mean } \\
(\mathrm{se}))\end{array}$ \\
\hline \multirow[t]{2}{*}{ All food } & Low-income (high-income) & $70(3)^{\star \star \star}$ & $79(2.76)^{\star \star \star}$ & $74(2.84)^{\star \star \star}$ \\
\hline & Māori (non-Māori) & $78(3.4)^{\star \star \star}$ & $79(3.69)^{\star \star \star}$ & $90(3.69)^{\star *}$ \\
\hline \multirow{2}{*}{$\begin{array}{l}\text { Fruit and } \\
\text { vegetables }\end{array}$} & Low-income (high-income) & $81(4.19)^{\star \star \star}$ & $84(3.93)^{\star \star \star}$ & $83(3.59)^{\star \star \star}$ \\
\hline & Māori (non-Māori) & $57(4.72)^{\star \star \star}$ & $63(4.38)^{\star \star \star}$ & $72(4.97)^{\star \star \star}$ \\
\hline \multirow[t]{2}{*}{ Nuts and seeds } & Low-income (high-income) & $76(11.08)^{\star \star \star}$ & $73(12.08)^{\star \star \star}$ & $68(9.98)^{\star \star \star}$ \\
\hline & Māori (non-Māori) & $40(11.34)^{\star \star \star}$ & $45(12.29)^{\star \star \star}$ & $55(11.23)^{\star \star \star}$ \\
\hline \multirow[t]{2}{*}{ Processed meat } & Low-income (high-income) & $84(4.74)^{\star \star \star}$ & $91(4.8)^{* *}$ & $91(4.97)^{*}$ \\
\hline & Māori (non-Māori) & $92(5.73)$ & $92(6.34)$ & $94(6.07)$ \\
\hline \multirow[t]{2}{*}{ Healthy foods } & Low-income (high-income) & $85(3.52)^{\star \star \star}$ & $88(3.48)^{\star \star \star}$ & $87(3.29)^{\star \star \star}$ \\
\hline & Māori (non-Māori) & $64(4.34)^{\star \star \star}$ & $65(3.77)^{\star \star \star}$ & $73(4.7)^{\star \star \star}$ \\
\hline \multirow{2}{*}{$\begin{array}{l}\text { Remaining less } \\
\text { healthy foods }\end{array}$} & Low-income (high-income) & $67(3.26)^{\star \star \star}$ & $78(2.98)^{\star \star \star}$ & $71(3.1)^{\star \star \star}$ \\
\hline & Māori (non-Māori) & $81(3.61)^{\star \star \star}$ & $83(4.08)^{\star \star \star}$ & $94(4.1)$ \\
\hline
\end{tabular}

\section{Proportions of household food group expenditure out of total food expenditure}

Table 4 presents proportion of specific food group expenditure out of tota/ household food expenditure by income-level and ethnicity for three HES waves. Overall, expenditure on fruit and vegetables accounted for $11 \%$ of the total food expenditure, less than $1 \%$ for nuts and seeds, $5 \%$ for processed meat, with $19 \%$ towards healthy foods and $81 \%$ for less healthy foods. There were small fluctuations in the proportions of specific food group expenditure out of total food expenditure, however, except for expenditure on nuts 
and seeds by Māori households (increased from 0.34\% in 2006/07 to 0.49\% in 2012/13), these changes were not statistically significant. 
Table 4

Means of proportion of specific food expenditure out of total annual household food expenditure by food group, income-level and ethnicity in three HES waves: 2006/07, 2009/10, and 2013/13.

\begin{tabular}{|c|c|c|c|c|}
\hline \multirow[t]{2}{*}{ Food group } & \multirow[t]{2}{*}{$\begin{array}{l}\text { Population } \\
\text { group }\end{array}$} & \multicolumn{3}{|c|}{$\begin{array}{l}\text { Proportion of food specific expenditure out of total food } \\
\text { expenditure }(\% ; \text { mean }(\mathrm{se}))\end{array}$} \\
\hline & & $2006 / 07$ & $2009 / 10$ & 2012/13 \\
\hline \multirow[t]{5}{*}{ Fruit and vegetables } & $\begin{array}{l}\text { Total } \\
\text { sample }\end{array}$ & $11.1(0.20)$ & $10.1(0.17)$ & $10.8(0.21)$ \\
\hline & $\begin{array}{l}\text { Low- } \\
\text { income }\end{array}$ & $11.8(0.28)$ & $10.3(0.23)$ & $11.2(0.30)$ \\
\hline & $\begin{array}{l}\text { High- } \\
\text { income }\end{array}$ & $10.2(0.25)$ & $9.8(0.23)$ & $10.2(0.23)$ \\
\hline & Māori & $8.1(0.41)$ & $8.4(0.32)$ & $8.9(0.43)$ \\
\hline & Non-Māori & $11.9(0.23)$ & $10.5(0.19)$ & $11.3(0.23)$ \\
\hline \multirow[t]{5}{*}{ Nuts and seeds } & $\begin{array}{l}\text { Total } \\
\text { sample }\end{array}$ & $0.63(0.03)$ & $0.77(0.04)$ & $0.79(0.04)$ \\
\hline & $\begin{array}{l}\text { Low- } \\
\text { income }\end{array}$ & $0.61(0.05)$ & $0.74(0.05)$ & $0.75(0.06)$ \\
\hline & $\begin{array}{l}\text { High- } \\
\text { income }\end{array}$ & $0.67(0.04)$ & $0.81(0.05)$ & $0.86(0.05)$ \\
\hline & Māori & $0.34(0.05)^{\star \star \star}$ & $0.46(0.06)^{\star \star \star}$ & $0.49(0.06)^{* \star \star}$ \\
\hline & Non-Māori & $0.71(0.04)$ & $0.85(0.04)$ & $0.87(0.04)$ \\
\hline \multirow[t]{5}{*}{ Processed meat } & $\begin{array}{l}\text { Total } \\
\text { sample }\end{array}$ & $5.45(0.13)$ & $5.21(0.11)$ & $5.14(0.12)$ \\
\hline & $\begin{array}{l}\text { Low- } \\
\text { income }\end{array}$ & $5.77(0.20)$ & $5.54(0.16)$ & $5.62(0.17)$ \\
\hline & $\begin{array}{l}\text { High- } \\
\text { income }\end{array}$ & $5.01(0.15)$ & $4.73(0.13)$ & $4.42(0.15)$ \\
\hline & Māori & $6.73(0.40)$ & $5.88(0.28)$ & $5.42(0.29)$ \\
\hline & Non-Māori & $5.14(0.12)$ & $5.04(0.12)$ & $5.08(0.13)$ \\
\hline \multirow[t]{3}{*}{ Healthy foods } & $\begin{array}{l}\text { Total } \\
\text { sample }\end{array}$ & $19.5(0.29)$ & $18.3(0.25)$ & $18.8(0.27)$ \\
\hline & $\begin{array}{l}\text { Low- } \\
\text { income }\end{array}$ & $21.2(0.41)$ & $19.3(0.33)$ & $20.0(0.38)$ \\
\hline & $\begin{array}{l}\text { High- } \\
\text { income }\end{array}$ & $17.2(0.35)$ & $16.9(0.35)$ & $16.9(0.32)$ \\
\hline
\end{tabular}

Note: ${ }^{*}{ }^{* *},{ }^{* * *}$ Denote statistical significance at the $10 \%, 5 \%$ and $1 \%$ levels, respectively. 


\begin{tabular}{|lllll|}
\hline & Māori & $16.0(0.55)$ & $16.1(0.48)$ & $16.0(0.66)$ \\
\cline { 2 - 5 } & Non-Māori & $20.4(0.34)$ & $18.9(0.28)$ & $19.5(0.29)$ \\
\hline $\begin{array}{l}\text { Remaining less } \\
\text { healthy foods }\end{array}$ & $\begin{array}{l}\text { Total } \\
\text { sample }\end{array}$ & $80.4(0.29)$ & $81.6(0.25)$ & $81.1(0.27)$ \\
\hline $\begin{array}{l}\text { Low- } \\
\text { income }\end{array}$ & $78.7(0.41)$ & $80.7(0.33)$ & $79.9(0.38)$ \\
\hline $\begin{array}{l}\text { High- } \\
\text { income }\end{array}$ & $82.7(0.35)$ & $83.0(0.35)$ & $83.0(0.32)$ \\
\hline $\begin{array}{ll}\text { Māori } \\
\text { Non-Māori }\end{array}$ & $83.9(0.55)$ & $83.8(0.48)$ & $83.9(0.66)$ \\
\hline Note: ${ }^{*},{ }^{* *},{ }^{* * *}$ Denote statistical significance at the 10\%, 5\% and 1\% levels, respectively. \\
\hline
\end{tabular}

Table 5 compares differences in the above expenditure proportions by income-level and ethnicity for each time-period. Low-income households spent greater proportions of their food budget on fruit and vegetables, processed meat and healthy foods than high-income households (peaking at $27 \%$ more than high-income households). Māori households spent greater proportions of the food budget on less healthy food especially in the first time period ( $30 \%$ more on processed meat and around $5 \%$ more on less healthy food in 2006/07) compared to non-Māori households; and a lower proportion of the food budget on healthy food ( $68 \%$ fruit and vegetables, $49 \%$ nuts and seeds, and $80 \%$ for healthy foods in $2006 / 07$ of the level in non-Māori households). These patterns largely persisted over the years, but with some nutritionfavourable trends for Māori households (eg, increased proportion on fruit and vegetables and decreased proportion on processed meat). 
Table 5

Relative risks (\%) in proportion of household food purchase expenditure for low-income as proportion of high-income and Māori as proportion of non-Māori households, HES 2006/07, 2009/10, and 2012/13.

\begin{tabular}{|c|c|c|c|c|}
\hline \multirow[t]{2}{*}{ Food group } & \multirow[t]{2}{*}{$\begin{array}{l}\text { Population group (as a } \\
\text { proportion of comparator) }\end{array}$} & \multicolumn{3}{|c|}{$\begin{array}{l}\text { Relative risks in proportion of food group expenditure } \\
\text { out of total food expenditure (\%: mean (se)) }\end{array}$} \\
\hline & & $2006 / 07$ & 2009/10 & $2012 / 13$ \\
\hline \multirow[t]{2}{*}{$\begin{array}{l}\text { Fruit and } \\
\text { vegetables }\end{array}$} & $\begin{array}{l}\text { Low-income (high- } \\
\text { income) }\end{array}$ & $116(3.33)^{\star \star \star}$ & $106(3.18)^{\star}$ & 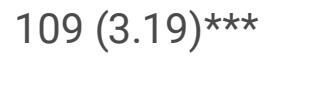 \\
\hline & Māori (non-Māori) & $68(4.76)^{\star \star \star}$ & $80(3.8)^{\star \star \star}$ & $78(4.57)^{\star \star \star \star}$ \\
\hline \multirow[t]{2}{*}{$\begin{array}{l}\text { Nuts and } \\
\text { seeds }\end{array}$} & $\begin{array}{l}\text { Low-income (high- } \\
\text { income) }\end{array}$ & $91(9.97)$ & $91(9.83)$ & $89(9.54)$ \\
\hline & Māori (non-Māori) & $49(11.26)^{\star \star \star}$ & $55(11.34)^{\star \star \star}$ & $56(10.52)^{\star \star \star}$ \\
\hline \multirow[t]{2}{*}{$\begin{array}{l}\text { Processed } \\
\text { meat }\end{array}$} & $\begin{array}{l}\text { Low-income (high- } \\
\text { income) }\end{array}$ & $114(4.51)^{\star \star \star}$ & $117(3.72)^{\star \star \star}$ & 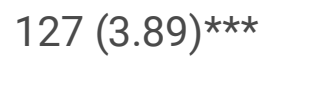 \\
\hline & Māori (non-Māori) & $130(7.5)^{\star \star \star}$ & $117(5.6)^{\star \star \star}$ & $106(5.84)$ \\
\hline \multirow[t]{2}{*}{ Healthy foods } & $\begin{array}{l}\text { Low-income (high- } \\
\text { income) }\end{array}$ & $124(2.6)^{\star \star \star}$ & $113(2.42)^{\star \star \star}$ & $118(2.42)^{\star \star \star}$ \\
\hline & Māori (non-Māori) & $80(3.73)^{\star \star \star}$ & $85(3.09)^{\star \star \star}$ & $82(3.89)^{\star \star \star}$ \\
\hline \multirow{2}{*}{$\begin{array}{l}\text { Remaining } \\
\text { less healthy } \\
\text { foods }\end{array}$} & $\begin{array}{l}\text { Low-income (high- } \\
\text { income) }\end{array}$ & $95(0.66)^{\star \star \star}$ & $97(0.56)^{\star \star \star}$ & $96(0.58)^{\star \star \star}$ \\
\hline & Māori (non-Māori) & $105(0.85)^{\star \star \star *}$ & $103(0.66)^{\star \star \star}$ & $104(0.86)^{\star \star \star}$ \\
\hline
\end{tabular}

\section{Discussion}

This analysis identified some nutritionally-favourable expenditure trends for Māori. There was increased expenditure on fruit and vegetables, nuts and seeds, and on the healthy food category in Māori households, although expenditure also increased on less healthy foods as well. As a result, the relative gap in health-related food expenditure between Māori and non-Māori households declined over time. However, a stark difference in expenditure remained, with around half to a quarter lower expenditure by Māori households in the healthy food groups. The trends in fruit and vegetables and nuts and seeds were similar for expenditure as a proportion of the food budget and as a proportion of income; although there was no significant change in these indicators for Māori expenditure on healthy foods. Some findings were less favourable from a nutritional perspective. There was little change in processed meat expenditure for Māori households and it remained at a level just slightly less than non-Māori households. 
Less healthy food expenditure as a proportion of total food expenditure changed little over time, but as a proportion of income it increased in Māori households more than non-Māori households (see Appendix B). Māori households differed significantly in income, household size and the percentage of households with children, so these factors may mediate the association between ethnicity and food expenditure.

Income inequities in food expenditure appeared to be relatively stable over time, however there were some potentially concerning trends from a nutrition perspective (unadjusted for ethnicity). High-income households experienced a greater increase in nuts and seeds expenditure (increasing the gap). Lowincome households had a greater increase in less healthy food expenditure, but it remained almost a third less than high-income households. Higher-income households also had a greater reduction in expenditure on processed meat (reducing the gap because expenditure remained higher than for low-income households).

Approximately $60 \%$ of the food available in NZ supermarkets have been classified as 'ultra-processed'. (27) In our study, we found roughly $81 \%$ of total food expenditure was spent on less healthy foods (Table 4 ). A diet that meets the NZ food and nutrition guidelines was estimated to cost approximately an extra $\$ 13.50$ per week for a family of four. ${ }^{(28)}$ It has been estimated for families on income support in NZ or on low wages, a healthy diet is unaffordable as it may cost up to a half of total household expenditure. ${ }^{(28)}$ However, our study found that on average, low-income households spent approximately $17 \%$ of their total income on food, including $3 \%$ on health foods and the rest on less healthy foods (Appendix B, Table B1). NZ's grocery prices are also relatively high overall. In 2017 NZ ranked as the sixth highest priced grocery market in the OECD, ${ }^{(29)}$ while its income per capita is below the OECD average. ${ }^{\left({ }^{30}\right)}$ New Zealanders also appear to spend a large proportion of their income on food and groceries. ${ }^{(29)}$ The country's 'supermarket duopoly' may be a contributing factor and this currently being investigated by the relevant NZ Government agency: the Commerce Commission.

\section{Strengths and limitations}

This study was the first (that we know of) to use HES data for informing food expenditure patterns in the NZ setting. Strengths of the HES data are that it is repeated every three years, is representative of the whole NZ population, and is a validated tool for informing economic policy. However, it should be noted that even though the Māori sample is at least large enough to perform the analysis, it does not provide equal explanatory power for Māori compared to non-Māori. These data included two-week diary for household expenditure so it reasonably covers the major food groups. However, there were some minor food category changes over the years in HES. There were also no data on non-commercial sources of food eg, home-grown, food from the wild (gathering kai moana [eg, shellfish], fishing, and hunting), gifting of food (a feature of both Māori and Pasifika cultures), and provision of free food via school breakfast and lunch programmes (for low-income schools). Nevertheless, these are not typically large sources of food for most people in NZ. Most importantly, there was no food purchase quantity data collected in this 
dataset and no accounting for food wastage (which can often be large in the fruit and vegetable category $\left.{ }^{(31)}\right)$.

\section{Research and policy implications}

From a health and nutritional perspective there is a need to keep the HES and have a routine plan to analyse the food expenditure aspects. But it is also desirable to further contextualise the HES data with other routine data collection (eg, supermarket sales data - albeit somewhat expensive to purchase from commercial providers). Also having regular adult and child nutrition surveys would be even better.

Policy options that the NZ Government could consider to improve nutrition and reduce inequities in dietrelated diseases include:

1. (a) Introducing subsidies for healthy food (eg, provision of vouchers for purchasing discounted fruit and vegetables from farmers markets in low-income communities). ${ }^{(32) ;}$

2. (b) Making unhealthy food more expensive (eg, via food taxes ${ }^{(25)}$. While such taxes could put more financial burden on low-income households, this can be addressed by using tax revenue to subsidise healthy food and expanding food in school programmes. In addition, tax revenue could be used to subsidise farmers markets in more deprived areas.

3. (c) Increasing the regulation around the marketing of unhealthy food (eg, especially marketing children are exposed to).

4. (d) Improving the nutrition-related labelling of foods and mandating warning labels on unhealthy foods (as used successfully in tobacco control, and being legalised in Chile ${ }^{(33)}$ ).

5. (e) Supporting culturally appropriate Māori led interventions to improve food security and healthy eating in Māori ${ }^{(34,35)}$ including protecting wild-food resources from waterway and marine pollution.

\section{Conclusions}

In this study HES data were useful for understanding trends in food expenditure patterns, but limitations remain and further investment in nutrition survey data is recommended. There seems to be slow improvements in diet inequalities by ethnicity, and no evidence of any improvement by income, implying much more must be done to address nutrition to reduce the burden of NCDs and NCD-related inequities in this high-income country.

\section{Abbreviations}

CPI: Consumer Price Index

CVD: Cardiovascular disease

DALYs: Disability-adjusted life years 
GBD: Global Burden of Disease

HES: Household Economic Survey

NZ: Aotearoa New Zealand

NCDs: Non-communicable diseases

OECD: Organisation for Economic Co-operation and Development

\section{Declarations}

\section{Ethics Approval}

Ethics Committee reference number HD19/057 by University of Otago, New Zealand.

\section{Consent for publication: N/A}

Availability of data and materials: Access to the anonymised data used in this study was provided by Statistics NZ under the security and confidentiality provisions of the Statistics Act 1975. Only people authorised by the Statistics Act 1975 are allowed to see data about a particular person, household, business, or organisation, and the results in this paper have been confidentialised to protect these groups from identification and to keep their data safe.

Competing interests: None

Funding: University of Otago Research Grant 2020

Authors' contributions: NN, AT, CC, NW designed the study. NN, AT performed data analyses. NN, AT, CC, $\mathrm{CM}$, NW interpreted the results. NN, AT wrote the first draft, and all authors contributed to the writing and approved the final version of the manuscript.

\section{Acknowledgements}

We thank Anja Mizdrak, Tony Blakely, Cliona Ni Mhurchu, Helen Eyles, and James Stanley for advice at early stages of this project.

\section{Disclaimer}

Access to the data used in this study was provided by Stats NZ under conditions designed to give effect to the security and confidentiality provisions of the Statistics Act 1975 . The results presented in this study are the work of the authors, not Stats NZ or individual data suppliers.

\section{References}


1. Afshin A, Sur PJ, Fay KA, Cornaby L, Ferrara G, Salama JS, et al. Health effects of dietary risks in 195 countries, 1990-2017: a systematic analysis for the Global Burden of Disease Study 2017. The Lancet. 2019;393(10184):1958-72.

2. Wang $\mathrm{H}$, Naghavi M, Allen C, Barber RM, Bhutta ZA, Carter A, et al. Global, regional, and national life expectancy, all-cause mortality, and cause-specific mortality for 249 causes of death, 1980-2015: a systematic analysis for the Global Burden of Disease Study 2015. The lancet. 2016;388(10053):1459-544.

3. Humphries DL, Dearden KA, Crookston BT, Woldehanna T, Penny ME, Behrman JR. Household food group expenditure patterns are associated with child anthropometry at ages 5, 8 and 12 years in Ethiopia, India, Peru and Vietnam. Economics \& Human Biology. 2017;26:30-41.

4. Fiedler JL, Carletto C, Dupriez O. Still Waiting for Godot? Improving Household Consumption and Expenditures Surveys (HCES) to Enable More Evidence-Based Nutrition Policies. Food and Nutrition Bulletin. 2012;33(3_suppl2):S242-S51.

5. Vandevijvere S, Monteiro C, Krebs-Smith SM, Lee A, Swinburn B, Kelly B, et al. Monitoring and benchmarking population diet quality globally: a step-wise approach. Obesity Reviews. 2013;14(S1):135-49.

6. Coates J, Rogers BL, Blau A, Lauer J, Roba A. Filling a dietary data gap? Validation of the adult male equivalent method of estimating individual nutrient intakes from household-level data in Ethiopia and Bangladesh. Food Policy. 2017;72:27-42.

7. Fiedler JL, Lividini K. Monitoring population diet quality and nutrition status with household consumption and expenditure surveys: suggestions for a Bangladesh baseline. Food Security. 2017;9(1):63-88.

8. Ishida A, Law S-H, Aita Y. Changes in food consumption expenditure in Malaysia. Agribusiness. 2003;19(1):61-76.

9. Sahal Estimé M, Lutz B, Strobel F. Trade as a structural driver of dietary risk factors for noncommunicable diseases in the Pacific: an analysis of household income and expenditure survey data. Globalization and Health. 2014;10(1):48.

10. Smart JC, Tschirley D, Smart F. Diet Quality and Urbanization in Mozambique. Food and Nutrition Bulletin. 2020;41(3):298-317.

11. Waid JL, Ali M, Thilsted SH, Gabrysch S. Dietary change in Bangladesh from 1985 to 2010. Global Food Security. 2018;17:221-32.

12. Russell J, Lechner A, Hanich Q, Delisle A, Campbell B, Charlton K. Assessing food security using household consumption expenditure surveys (HCES): a scoping literature review. Public Health Nutrition. 2018;21(12):2200-10.

13. Melgar-Quinonez HR, Zubieta AC, MkNelly B, Nteziyaremye A, Gerardo MFD, Dunford C. Household Food Insecurity and Food Expenditure in Bolivia, Burkina Faso, and the Philippines. The Journal of Nutrition. 2006;136(5):1431S-7S. 
14. Kirkpatrick S, Tarasuk V. The relationship between low income and household food expenditure patterns in Canada. Public Health Nutrition. 2003;6(6):589-97.

15. Teng AM, Atkinson J, Disney G, Wilson N, Sarfati D, McLeod M, et al. Ethnic inequalities in cancer incidence and mortality: census-linked cohort studies with 87 million years of person-time follow-up. BMC Cancer. 2016;16(1):755.

16. Disney G, Teng A, Atkinson J, Wilson N, Blakely T. Changing ethnic inequalities in mortality in New Zealand over 30 years: linked cohort studies with 68.9 million person-years of follow-up. Population Health Metrics. 2017;15(1):15.

17. Nghiem N, Blakely T, Cobiac LJ, Cleghorn CL, Wilson N. The health gains and cost savings of dietary salt reduction interventions, with equity and age distributional aspects. BMC Public Health. 2016;16(1).

18. Health Central. Is it time for an updated nutrition survey in NZ? 2019 [Available from: https://healthcentral.nz/is-it-time-for-an-updated-nutrition-survey-in-nz/.

19. Stats NZ. Integrated Data Infrastructure (IDI) 2019 [Available from: http://archive.stats.govt.nz/browse_for_stats/snapshots-of-nz/integrated-data-infrastructure/ididata.aspx.

20. StatsNZ. Expanding the Household Economic Survey to obtain good measures of child poverty 2019 [Available from: https://www.stats.govt.nz/methods/expanding-the-household-economic-survey-toobtain-good-measures-of-child-poverty.

21. Carletto C, Zezza A, Banerjee R. Towards better measurement of household food security: Harmonizing indicators and the role of household surveys. Global Food Security. 2013;2(1):30-40.

22. Ball C, Ormsby J. Comparing the Household Economic Survey to administrative records: An analysis of income and benefit receipt (AP 17/01). The Treasury; 2017.

23. Ni Mhurchu C, Eyles H, Schilling C, Yang Q, Kaye-Blake W, Genç M, et al. Food Prices and Consumer Demand: Differences across Income Levels and Ethnic Groups. PLOS ONE. 2013;8(10):e75934.

24. Nghiem N, Jacobi L, Ramirez Hassan A, Waterlander W, Blakely T. Thomas Bayes goes to the Virtual Supermarket: Combining prior food price elasticities and experimental data in a large demand system of dietary choices. Available at SSRN 3252225. 2018.

25. Waterlander WE, Jiang Y, Nghiem N, Eyles H, Wilson N, Cleghorn C, et al. The effect of food price changes on consumer purchases: a randomised experiment. The Lancet Public Health. 2019;4(8):e394-e405.

26. Waterlander WE, Blakely T, Nghiem N, Cleghorn CL, Eyles H, Genc M, et al. Study protocol: combining experimental methods, econometrics and simulation modelling to determine price elasticities for studying food taxes and subsidies (The Price ExaM Study). BMC Public Health. 2016;16(1):601.

27. Mackay S, Ni Mhurchu C, Swinburn B, Eyles H, Young L, Gontijo de Castro T. State of the food supply: New Zealand 2019. 2019.

28. Vandevijvere S, Mackay S, D'Souza E, Swinburn B. How healthy are New Zealand food environments? A comprehensive assessment 2014-2017: The University of Auckland; 2020. 
29. The New Zealand Commerce Commission Te Komihana Tauhokohoko. Market study into the retail grocery sector - Draft Report. URL:

https://comcom.govt.nz/_data/assets/pdf_file/0025/260377/Market-study-into-the-retail-grocerysector-Draft-report-29-July-2021.pdf. Wellington; 2021.

30. OECD. Gross Domestic Product GDP. URL: https://data.oecd.org/gdp/gross-domestic-productgdp.htm 2021 [

31. Quested T, Johnson H. Household food and drink waste in the UK: Wastes \& Resources Action Programme (WRAP); 2009.

32. Bowers S, Carter K, Gorton D, Heta C, Lanumata T, Maddison R, et al. Enhancing food security and physical activity for Māori, Pacific and low-income peoples. August 2009. Wellington: Clinical Trials Research Unit, University of Auckland; GeoHealth Laboratory, University of Canterbury. Health Promotion and Policy Research Unit, University of Otago. 2009.

33. Hall MG, Grummon AH. Nutrient Warnings on Unhealthy Foods. JAMA. 2020;324(16):1609-10.

34. RNZ News. 'Uncertain times' lead to kai network in Auckland. URL: https://www.rnz.co.nz/news/temanu-korihi/450455/uncertain-times-lead-to-kai-network-in-auckland 2021 [

35. Hutchings J. Te Mahi Māra Hua Parakore A Māori Food Sovereignty Handbook. Ōtaki, New Zealand: Te Tākapu, Te Wānanga o Raukawa; 2015.

\section{Supplementary Files}

This is a list of supplementary files associated with this preprint. Click to download.

- Appendix.docx 